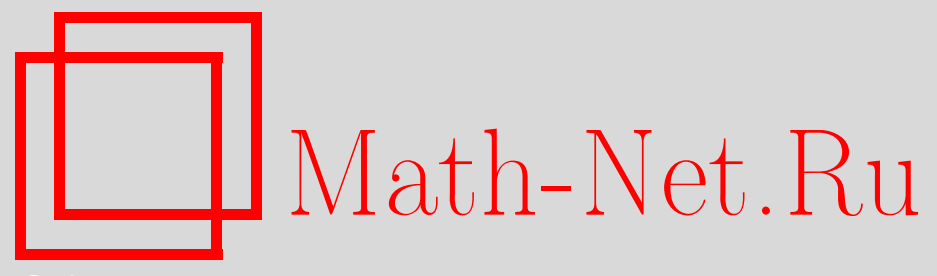

В. И. Сенашов, В. П. Шунков, Почти слойная конечность периодической части группы без инволюций, Дискрет. матем., 2003, том 15, выпуск 3, 91-104

DOI: https://doi.org/10.4213/dm208

Использование Общероссийского математического портала Math-Net.Ru подразумевает, что вы прочитали и согласны с пользовательским соглашением http://www . mathnet.ru/rus/agreement

Параметры загрузки:

IP: 54.92 .164 .108

26 апреля 2023 г., 16:47:01

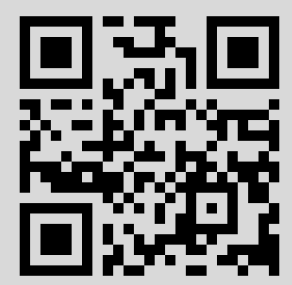




\title{
Почти слойная конечность периодической части группы без инволюций
}

\author{
() 2003 г. В. И. Сенашов, В. П. Шунков
}

\begin{abstract}
В работе доказана следующая теорема, характеризующая в классе групп без инволюций класс групп, обладающих почти слойно конечной периодической частью: если в группе Шункова без инволюций нормализатор любой нетривиальной конечной подгруппы обладает почти слойно конечной периодической частью, то и сама группа обладает почти слойно конечной периодической частью.

Работа выполнена при поддержке Российского фонда фундаментальных исследований, проект 99-01-00432, и при поддержке гранта №9 6-го конкурса-экспертизы 1999 г. научных проектов молодых ученых РАН.
\end{abstract}

\section{1. Введение}

В работе доказана следующая теорема, характеризующая в классе групп без инволюций класс групп, обладающих почти слойно конечной периодической частью: если в группе Шункова без инволюций нормализатор любой нетривиальной конечной подгруппы обладает почти слойно конечной периодической частью, то и сама группа обладает почти слойно конечной периодической частью. Теорема является обобщением на смешанные группы результатов работ автора $[1,2]$.

Условие для группы быть группой Шункова в этой теореме отбросить нельзя ввиду примеров групп Новикова-Адяна [3] и Ольшанского [4]. Напомним, что группа $G$ называется группой Шункова, если для любой конечной подгруппы $H$ в факторгруппе $N_{G}(H) / H$ любые два сопряженных элемента простого порядка порождают конечную подгруппу.

Напомним некоторые определения, которые будут нам необходимы в дальнейшем.

Группа называется слойно конечной, если множество ее элементов любого данного порядка конечно [5].

Группа называется почти слойно конечной, если она является конечным расширением слойно конечной группы. Очевидно, черниковская группа является почти слойно конечной.

Группа называется черниковской, если она либо конечна, либо является конечным расширением прямого произведения конечного числа квазициклических групп [6, 7].

Локально конечная группа $G$ называется $S F$-группой, если для любого $p \in \pi(G)$ силовские $p$-подгруппы из $G$ суть черниковские группы [8].

Группа называется локально нормальной, если в ней любое конечное множество элементов содержится в конечной подгруппе, нормальной в самой группе [9]. 


\section{2. Известные результаты}

В этом разделе для удобства чтения статьи мы приведем известные результаты, на которые в дальнейшем будем ссылаться как на предложения с соответствующим номером.

Предложение 1. Если в локально конечной группе $G$ для некоторого $p \in \pi(G)$ существует конечная силовская p-подгруппа, то все силовские p-подгруппы из $G$ конечны и сопряжены в $G$ между собой.

Доказательство этого предложения легко получить, используя локальную конечность группы $G$ и теорему Силова [6].

Предложение 2 (лемма Фраттини). Пусть $G$ - локально конечная группа, $T$ - нормальная подгруппа в $G$ u $S$ - некоторая конечная силовская p-подгруппа из $T$. Тогда $G=T N_{G}(S)$.

Доказательство этой леммы легко получить, опираясь на предложение 1.

Предложение 3. Группа является слойно конечной, если она локально нормальна и является SF-групnой.

Доказательство см. в $[5,10]$.

Предложение 4. В черниковской р-группе любая максимальная элементарная абелева подгруппа конечна.

Это утверждение непосредственно вытекает из определения черниковской группы.

Предложение 5 (теорема Блекберна). Если в локально конечной р-группе некоторая максимальная элементарная абелева подгруппа конечна, то и сама группа черниковская.

Доказательство см. в $[5,11]$.

Предложение 6 (теорема Каргаполова). Всякая почти локально разрешимая SF-группа обладает полной частью.

Доказательство см. в [12].

Предложение 7. SF-группа почти локально разрешима и обладает полной частью.

Это утверждение вытекает из теоремы, доказанной в [13], и предположения 6.

Предложение 8 (теорема Горчакова). Всякая периодическая почти локально разрешимая группа, в которой ранги абелевых подгрупп конечны, имеет конечный ранг.

Доказательство см. в [14].

Предложение 9 (теорема Каргаполова). В каждой локально разрешимой группе конечного ранга содержится подгруппа конечного индекса, коммутант которой обладает возрастаючим чентральным рядом.

Доказательство см. в [15].

Предложение 10 (теорема Черникова). Если $G$ - периодическая локально разрешимая группа и в ней по некоторому $p \in \pi(G)$ силовские p-подгруппы конечны, то индекс $\left|G: O_{p^{\prime}}(G)\right|$ конечен. 
Доказательство см. в [5].

Предложение 11. Пусть $G-$ конечная группа вида $G=H \lambda L$, где $H-p^{\prime}$-nодгруппа, $L$ - элементарная абелева p-подгруппа порядка, не меньшего $p^{2}$. Тогда

$$
G=\operatorname{gr}\left(h \in C_{G}(t) \mid t \in R^{\#}=R \backslash\{1\}\right) .
$$

Доказательство см. в [16].

Предложение 12 (теорема Томпсона). Конечная группа, обладающая регулярным автоморфизмом простого порядка, нильпотента.

Доказательство см. в [17].

Предложение 13. В локально конечной группе с черниковскими силовскими подгруппами силовские р-подгруппы сопряжены.

Доказательство см. в [18].

Предложение 14. Если в разрешимой группе вида $B \lambda(a)$, где $B-p^{\prime}$-группа, $(a)-$ - иклическая группа порядка р, элемент а действует регулярно на нильпотентном радикале группы, то он действует регулярно и на группе $B$.

Доказательство см. в [19].

Предложение 15. Пусть $G$ - конечная группа вида $G=O_{p^{\prime}}(G) \lambda R$, где $R$ - элементарная абелева подгруппа порядка $p^{2}$. Тогда $O_{p^{\prime}}(G)$ содержится в подгруппе, порожденной чентрализаторами неединичных элементов из $R$.

Доказательство см. в [20].

Предложение 16. Периодическая почти локально разрешимая группа, обладающая элементом простого порядка с конечным чентрализатором, почти нильпотента.

Доказательство см. в [21].

Предложение 17. Пусть $G$ - группа Фробениуса вида $G=F \lambda(x)$, где х имеет простой порядок р. Предположим еще, что подгруппы $\operatorname{gr}\left(x, x^{g}\right)$ конечны для каждого $g \in G$ и что каждая абелева $(x)$-инвариантная подгруппа простой экспоненты из $F$ конечна. Тогда $F$ - нильпотентная группа.

См. лемму 4.6 в [22].

Предложение 18. Группа Шункова, удовлетворяющая условию минимальности для периодических абелевых подгрупп, обладает черниковской периодчческой частью.

Доказательство см. в [23].

Предложение 19. Пусть $G$ - группа, $H$ - ее собственная подгруппа, а - элемент простого порядка $p \neq 2$ из $G$ такие, что почти для всех (то есть для всех, кроме, быть может, конечного числа) элементов вида $g^{-1} a g$, где $g \in G \backslash H$, подгруппь $L_{g}=\operatorname{gr}\left(a, g^{-1} a g\right)$ являются группами Фробениуса с неинвариантным множителем (a). Тогда либо $G=F \lambda N_{G}(a)$ и $F \lambda(a)$ - группа Фробениуса с ядром $F$ и неинвариантным множителем (a), либо элемент а лежит в конечном нормальном делителе группь $G$. 
Доказательство см. в [24] (см. также [19]).

Предложение 20. Пусть $T$ - группа, $D$ - ее локально конечная подгруппа с черниковскими примарными подгруппами $A, C$ - некоторые подгруппы из $T$. Если $D$ обладает такими подгруппами $F, R, R \leqslant F$, что индекс $|D: R|$ конечен $u A, D<$ $N_{T}(F)$ и $C, D<N_{T}(R)$, то в $D$ cуществует подгруппа $X$ конечного индекса в $D u$ $A, C, D<N_{T}(X)$.

Доказательство см. в [18].

Предложение 21. В черниковской р-группе лишь конечное число несопряженных элементов имеет конечные чентрализаторы.

Доказательство см. в [25] (см. также [22]).

Предложение 22. Абелева р-группа тогда и только тогда является черниковской группой, когда ее нижний слой конечен.

Доказательство см., например, в [5].

Предложение 23 (теорема Черникова). В черниковской группе существует лишь конечное число несопряженных конечных разрешимых подгрупп заданного порядка.

Доказательство см., например, в [19].

\section{3. Характеризация почти слойно конечной группы в классе локально конечных групп}

Теорема 1. Локально конечная группа $G$ тогда и только тогда почти слойно конечна, когда в $G$ выполняется условие

(*) нормализатор любой нетривиальной конечной подгруппы из $G$ - почти слойно конечная группа.

Необходимость условия (*) очевидна. Пусть в $G$ выполняется условие $(*)$, докажем, что группа $G$ почти слойно конечна. Предварительно докажем несколько лемм, предполагая, что локально конечная группа $G$ с условием $(*)$ не является почти слойно конечной.

В этом разделе мы будем использовать обозначения: если $G-S F$-группа, то ее полную часть обозначим через $\widetilde{G}$, а слойно конечный радикал почти слойно конечной группы $H$ - через $\widehat{H}$.

Лемма 1. $G-S F$-груnna.

Доказательство. Пусть $Q$ - некоторая силовская $p$-подгруппа из $G$, где $p \in \pi(G)$. Если бы $Q$ не являлась черниковской подгруппой, то по предложению $5 Q$ обладала бы бесконечной элементарной абелевой подгруппой $A$. Взяв из $A$ элемент $a \neq 1$ и применив к $C_{Q}(a)$ условие $(*)$, мы заключили бы, что $C_{Q}(a)$ - почти слойно конечная гругпа. Но $Q$ - p-группа, а поэтому $C_{Q}(a)$ - черниковская подгруппа (см. предложение 3 ).

Однако это невозможно, так как $A<C_{Q}(a)$ и $A$ - бесконечная элементарная абелева подгруппа из $Q$ (предложение 4). Полученное противоречие означает, что в $Q$ любая элементарная абелева подгруппа конечна и по предложению $5 Q$ - черниковская группа. А так как $p-$ любое число из $\pi(G)$, этим самым мы доказали, что $G-S F$-группа. Лемма доказана. 
Лемма 2. G - почти локально разрешимая группа с конечными силовскими подгруппами.

Доказательство. По лемме $1 G-S F$-группа, а по предположению $7 G$ - почти локально разрешимая группа и $G$ обладает полной периодической частью $\widetilde{G}$. Если бы $\widetilde{G} \neq 1$, то, очевидно, $\widetilde{G}$ обладала бы нетривиальной конечной подгруппой $V$, нормальной в $G$, то есть $N_{G}(V)=G$. Но тогда по условию (*) группа $G$ почти слойно конечна вопреки ранее сделанному предположению. Следовательно, $\widetilde{G}=1$ и в $G$ силовские подгруппы конечны. Лемма доказана.

Јемма 3. Не нарушая общности, можн предполагать, что группа $G$ локально разреиима и имеет вид $G=E \lambda R$, где $R$ - элементарная абелева р-подгруппа порядка $p^{3}$ и $E-p^{\prime}$-nodzpynna.

Доказательство. По лемме $2 G$ - почти локально разрешимая группа, а это означает, что $G$ обладает локально разрешимой подгруппой $B$, нормальной в $G$ и с конечным индексом $|G: B|$. Очевидно, что если бы подгруппа $B$ была почти слойно конечной, то группа $G$ являлась бы также почти слойно конечной вопреки предположению. Следовательно, $B$ не есть почти слойно конечная группа. Но тогда, не нарушая общности рассуждений, будем предполагать, что $G=B$, то есть группа $G$ локально разрешима.

Если бы в $G$ для любого $q \in \pi(G)$ силовская $q$-группа из $G$ не обладала элементарной абелевой подгруппой порядка $q^{3}$, то, очевидно, ранг любой абелевой подгруппы из $G$ (ранг в смысле А. И. Мальцева) не превосходил бы двух. В этом случае по предложению 8 $G$ имеет конечный ранг, а ввиду предложения $9 G$ обладает нормальной подгруппой $V$ с конечным $|G: V|$ и такой, что ее коммутант $V^{\prime}=[V . V]-$ локально нильпотентная группа. Если $V^{\prime}=1$, то есть $V$ - абелева подгруппа, или $V^{\prime} \neq 1$, то в обеих ситуациях группа $G$, очевидно, обладает нетривиальной конечной нормальной подгруппой. Но тогда по условию (*) группа $G$ была бы почти слойно конечной вопреки предположению.

Полученное противоречие означает, что для некоторого $p \in \pi(G)$ группа $G$ обладает элементарной абелевой подгруппой $R$ порядка $p^{3}$. Так как силовские $p$-подгруппы из $G$ конечны, то по предложению $10\left|G: O_{p^{\prime}}(g)\right|<\infty$. Очевидно, $E=O_{p^{\prime}}(G)$ не является почти слойно конечной и, не нарушая общности рассуждений, будем полагать, что $G=E \lambda R$. Лемма доказана.

Лемма 4. Если $\Sigma$ - некоторая локальная система подгруппы $E$, состоящая из конечных nодгрупп из $E$, то

$$
\bar{\Sigma}=\left\{\operatorname{gr}\left(X^{R}\right) \mid X \in \Sigma\right\}
$$

- локальная система подгруппы $E$, состоящая из конечных $R$-инвариантных подгрупп.

Доказательство очевидно.

В соответствии с леммой 4 в лемме 5 предполагается, что $\Sigma$ - локальная система подгруппы $E$, состоящая из конечных $R$-инвариантных подгрупп из $\Sigma$.

Лемма 5. Справедливо равенство $G=\operatorname{gr}\left(h \in C_{G}(t) \mid t \in R^{\#}\right)$.

Доказательство. Пусть $H-$ подгруппа из $\Sigma$. Как отмечено выше, $H-R$-инвариантная подгруппа и, кроме этого, $H-p^{\prime}$-подгруппа, поэтому $B=H \lambda R$. По предложению 11

$$
B<\operatorname{gr}\left(h \in C_{B}(t) \mid t \in R^{\#}\right) .
$$

Но $H$ - любая подгруппа из $\Sigma$, а так как $\Sigma$ - локальная система для подгруппы $E$, отсюда вытекает утверждение леммы. 
Лемма 6. Если $t \in R^{\#}$, то иентрализатор $C_{G}(t)$ бесконечен.

Доказательство. Предположим, что для некоторого $r \in R^{\#}$ централизатор $C_{G}(r)$ конечен. Пусть $q_{1}, q_{2}, \ldots, q_{m}$ - все простые делители порядка $\left|C_{G}(r)\right|$. По лемме $2 G-$ почти локально разрешимая группа с конечными силовскими подгруппами. Отсюда и из предложения 10 вытекает, что $G$ обладает нормальной $q_{i}^{\prime}$-подгруппой $V_{i}$ с конечной фактор-группой $G / V_{i}, i=1,2, \ldots, m$. Пусть

$$
V=\bigcap_{i=1}^{m} V_{i}
$$

Очевидно, $V-\pi^{\prime}$-подгруппа, где $\pi=\left\{q_{1}, q_{2}, \ldots, q_{m}\right\}$, и по теореме Пуанкаре [6] $V \triangleleft G$ и $|G: V|<\infty$. Очевидно, что $r$ индуцирует регулярный автоморфизм простого порядка $p$, и ввиду предложения $12 V$ - нильпотентная группа. Но тогда $G$ - почти слойно конечная группа вопреки предположению. Полученное противоречие означает, что $C_{G}(r)$ бесконечен, и лемма доказана.

Лемма 7. В группе $G$ существует подгруппа $V$, обладающая следующими свойствами:

(1) V- почти слойно конечная подгруппа;

(2) $R<V u\left|C_{G}(r): D_{r}\right|<\infty$, где $D_{r}=V \cap C_{G}(r), r \in R^{\#}$.

Доказательство. Пусть $r_{0}$ - элемент из $R^{\#}=R \backslash\{1\}$. Рассмотрим $V_{0}=C_{G}\left(r_{0}\right)$. По лемме $6 V_{0}$ бесконечна, а так как $|R| \geqslant p^{3}$, то ввиду предложения 11 и лемм 3 и 6 $R$ обладает подгруппой $X$ порядка, не меньшего $p^{2}$, и индексом $|R: X|=p$, причем централизатор $C_{V_{0}}(X)$ бесконечен.

Введем следующие обозначения. Пусть $Z=\widehat{V}_{0} \cap C_{V_{0}}(X),\left\{r_{0}, r_{2}, \ldots, r_{n}\right\}-$ все различные элементы из $X^{\#}$ и $B_{i}=C_{G}\left(r_{i}\right), i=0,1,2, \ldots, n$. Так как $\left|V_{0}: \widehat{V}_{0}\right|<\infty$ и $C_{V}(X)$ бесконечен, то, очевидно, что $Z$ - бесконечная $R$-инвариантная подгруппа и $Z<B_{i}$, $i=1,2, \ldots, n$. Пусть $Z_{1}=Z \cap \widehat{B}_{1}$, где $B_{1}=C_{G}\left(r_{1}\right)$. Так как $\widehat{B}_{1} \triangleleft B_{1},\left|B_{1}: \widehat{B}_{1}\right|<\infty, Z<B_{1}$ и $Z$ - бесконечная группа, то пересечение $Z_{1}$ также бесконечно, причем $R<N_{G}\left(Z_{1}\right)$. Далее, $Z_{1}$ - слойно конечная подгруппа и по лемме 3 локально разрешима. Поэтому $Z_{1}$ обладает нетривиальной $R$-инвариантной конечной $q_{1}$-подгруппой $Q_{1}$, нормальной в $Z_{1}$. Рассмотрим $V_{1}=N_{G}\left(Q_{1}\right)$. По условию $(*) V_{1}$ - почти слойно конечная подгруппа. Очевидно, индексы $\left|V_{0}: V_{1} \cap V_{0}\right|$ и $\left|B_{1}: V_{1} \cap B_{1}\right|$ конечны, где $B_{1}=C_{G}\left(r_{1}\right)$.

Так как $Z_{1}<V_{1} \cap B_{2}$, где $B_{2}=C_{G}\left(r_{2}\right),\left|V_{1}: \widehat{V}_{1}\right|<\infty,\left|B_{1}: \widehat{B}_{2}\right|<\infty$ и $Z_{1}-$ бесконечная группа, то пересечение $Z_{2}=\widehat{V}_{1} \cap \widehat{B}_{2} \cap Z_{1}$ также бесконечно и, очевидно, $R<N_{G}\left(Z_{2}\right)$. Далее, подгруппа $Z_{2}$ слойно конечна и по лемме 3 локально разрешима. Но тогда $Z_{2}$ обладает нетривиальной $R$-инвариантной конечной $q_{2}$-подгруппой $Q_{2}$, нормальной в $Z_{2}$. По условию (*) подгруппа $V_{2}=N_{G}\left(Q_{2}\right)$ почти слойно конечна, и, очевидно, индексы $\left|V_{1}: V_{2} \cap V_{1}\right|$ и $\left|B_{2}: V_{2} \cap B_{2}\right|$ конечны, где $B_{2}=C_{G}\left(r_{2}\right)$. Относительно элемента $r_{3}$ и подгруппы $V_{2}$ рассуждаем также, как и при рассмотрении пары $\left(r_{2}, V_{1}\right)$, и так далее.

Рассуждая таким образом, построим последовательность подгрупп

$$
V_{0}, V_{1}, V_{2}, \ldots, V_{n}
$$

где $V_{i}=N_{G}\left(Q_{i}\right), Q_{i}$ - конечная $R$-инвариантная $q_{i}$-подгруппа из $Z_{i}$ и $Q_{i} \triangleleft Z_{i}$, $i=1,2, \ldots, n$. На основании построения последовательности (1) заключаем, что индексы $\left|V_{i}: V_{i+1} \cap V_{i}\right|$ и $\left|B_{i+1}: V_{i+1} \cap B_{i+1}\right|, i=0,1, \ldots, n-1$, конечны, где $V_{0}=B_{0}=C_{G}\left(r_{0}\right)$. 
Рассмотрим множество

$$
R \backslash X=\left\{r_{n+1}, r_{n+2}, \ldots, r_{n+m}\right\}
$$

и централизаторы

$$
B_{n+1}=C_{G}\left(r_{n+1}\right), B_{n+2}=C_{G}\left(r_{n+2}\right), \ldots, B_{n+m}=C_{G}\left(r_{n+m}\right) .
$$

Ввиду предложения 11 и лемм $3,6 R$ обладает подгруппой $X_{i}$ такой, что $\left|R: X_{i}\right|=p$, $r_{i} \in X_{i}$ и централизатор $C_{B_{n+i}}\left(X_{i}\right)$ бесконечен, $i=1,2, \ldots, m$.

Так как $\left|B_{n+i}: \widehat{B}_{n+i}\right|<\infty$, пересечение $T_{i}=\widehat{B}_{n+i} \cap C_{B_{n+i}}\left(X_{i}\right)$ бесконечно, $i=1,2, \ldots, m$. Но поскольку $|R| \geqslant p^{3}$ и $|R: X|=\left|R: X_{i}\right|=p, i=1,2, \ldots, m$, по теореме Ремака [6] $X \cap X_{i} \neq 1, i=1,2, \ldots, m$.

Возьмем в пересечении $X \cap X_{i}$ некоторый нетривиальный элемент $t_{i}, i=1, \ldots, m$. В частности, $t_{1} \in X \cap X_{1}$ и $t_{1} \neq 1$, причем $T_{1}<C_{G}\left(t_{1}\right)$ и $T_{1}=\widehat{B}_{n+1} \cap C_{B_{n+1}}\left(X_{1}\right)-$ бесконечная подгруппа. Отсюда, опираясь на свойства последовательности (1), заключаем, что $W_{1}=T_{1} \cap \widehat{V}_{N}$ - бесконечная подгруппа и $R<N_{G}\left(W_{1}\right)$. По лемме $2 W_{1}$ обладает конечной силовской $q_{n+1}$-подгруппой $Q_{n+1}$, где $q_{n+1} \in \pi\left(W_{1}\right)$, причем ее можно выбрать так, что $R<N_{G}\left(Q_{n+1}\right)$ (см. предложения 1 и 2). Рассмотрим $V_{n+1}=N_{G}\left(Q_{n+1}\right)$. Так как $Q_{n+1}<\widehat{V}_{n} \cap \widehat{B}_{n+1}$, ввиду слойной конечности подгрупп $\widehat{V}_{n}, \widehat{B}_{n+1}$ (условие $\left.(*)\right)$ и конечности индексов $\left|V_{n}: \widehat{V}_{n}\right|$ и $\left|B_{n+1}: \widehat{B}_{n+1}\right|$, индексы $\left|V_{n}: V_{n+1} \cap V_{n}\right|$ и $\left|B_{n+1}: V_{n+1} \cap B_{n+1}\right|$ также конечны, и кроме этого, по условию (*) подгруппа $V_{n+1}$ почти слойно конечна. Относительно тройки $\left(V_{n+1}, X_{2}, B_{n+2}\right)$, рассуждая аналогично, построим почти слойно конечную подгруппу $V_{n+2}=N_{G}\left(Q_{n+2}\right)$, где $Q_{n+2}$ - силовская $q_{n+2}$-подгруппа из $W_{2}=T_{2} \cap \widehat{V}_{n+1}$ и $R<N_{G}\left(Q_{n+2}\right)=V_{n+2}, q_{n+2} \in \pi\left(W_{2}\right)$.

Так же, как и при рассмотрении тройки $\left(V_{n}, X_{1}, B_{n+1}\right)$, докажем конечность индексов $\left|V_{n+1}: V_{n+2} \cap V_{n+1}\right|,\left|B_{n+2}: V_{n+2} \cap B_{n+2}\right|$. Рассуждая таким образом для любого $i=2,3, \ldots, m$, построим почти слойно конечную подгруппу $V=V_{n+m}=N_{G}\left(Q_{n+m}\right)$, для которой индексы $\left|V_{n+m-1}: V_{n+m} \cap V_{n+m-1}\right|,\left|B_{n+m}: V_{n+m} \cap B_{n+m}\right|$ конечны. Очевидно, что подгруппа $V=V_{n+m}$ обладает свойствами (1) и (2) из леммы 7. Лемма доказана.

Перейдем непосредственно к доказательству теоремы 1. Ниже будем придерживаться обозначений, введенных в лемме 7 и в ее доказательстве, то есть

$$
V=V_{n+m}=N_{G}\left(Q_{n+m}\right)
$$

- группа, обладающая свойствами (1), (2) из леммы 7,

$$
R^{\#}=R \backslash\{1\}=\left\{r_{0}, r_{1}, r_{2}, \ldots, r_{n+m}\right\},
$$

$B_{i}=C_{G}\left(r_{i}\right), i=0,1,2, \ldots, n+m$.

По лемме $7 B_{i}$ - почти слойно конечная группа и индекс $\left|B_{i}: \widehat{V} \cap \widehat{B}_{i}\right|$ конечен, $i=0,1,2, \ldots, n+m$. Как известно [9], $\widehat{V} \cap \widehat{B}_{i}$ обладает подгруппой $A_{i}$ с конечным индексом, нормальной в $B_{i}, i=0,1,2, \ldots, n+m$. Очевидно, что $B_{i}=A_{i} S_{i}$, где $S_{i}-$ конечная подгруппа из $B_{i}$. Положим

$$
\pi_{i}=\pi\left(S_{i}\right), \quad i=0,1, \ldots, n=m, \quad \pi=\pi_{0} \cup \pi_{1} \cup \ldots \cup \pi_{i} \cup \ldots \cup \pi_{n+m} .
$$

Множество $\pi$ конечно, и ввиду лемм 2, 3, предложения 10 и теоремы Пуанкаре [9] группа $G=E \lambda R$ обладает $\pi^{\prime}$-подгруппой $L$ из $E$ с конечным индексом $|G: L|$, нормальной в

4 Дискретная математика, т.15 №3 
$G$, где $E-p^{\prime}$-подгруппа. Рассмотрим подгруппы $K=L \lambda R$ и $H=V \cap K$. Очевидно, что

$$
C_{K}\left(r_{i}\right) \leqslant A_{i} \lambda R \leqslant H, \quad i=0,1, \ldots, n+m .
$$

На основании леммы 5 заключаем, что $H=K$, то есть подгруппа $K$ почти слойно конечна. Так как $|G: K|<\infty$, то и $G$ - почти слойно конечная группа. Таким образом, в силу леммы 3 доказательство теоремы завершено.

\section{4. Характеризация группы с почти слойно конечной периодической частью}

Теорема 2. Пусть $G$ - группа Шункова без инволючий. Если в $G$ нормализатор любой нетривиальной конечной подгруппы обладает почти слойно конечной периодической частью, то и сама группа $G$ обладает почти слойно конечной периодической частью.

Проведем доказательство от противного, предположим, что теорема неверна и $G$ группа-контрпример. В этом предположении докажем для группы $G$ ряд лемм.

Лемма 8. Силовские p-подгруппы в $G$ черниковские, в частности, $G$ не может обладать периодической частью, являющейся р-группой.

Доказательство. Пусть $P$ - p-подгруппа группы $G$. Среди ее элементарных абелевых подгрупп, очевидно, найдется максимальная подгруппа $R$. Бесконечной группа $R$ быть не может ввиду условий теоремы. Следовательно, любая абелева подгруппа из $P$ имеет конечный нижний слой. Тогда по предложению 22 абелевы подгруппы удовлетворяют условию минимальности, и по предложению $18 P$ - черниковская группа. Если же $G$ является $p$-группой, то по только что доказанному она должна быть черниковской группой, что невозможно ввиду выбора контрпримера. Полученное противоречие доказывает лемму.

Лемма 9. Группа $G$ не обладает неединичным локально конечным радикалом.

Доказательство. Если локально конечный радикал группы $G$ неединичен, то он почти слойно конечен по условию доказываемой теоремы и в силу теоремы 1 , следовательно, в нем найдется конечная характеристическая подгруппа, нормализатор которой по условию теоремы обладает почти слойно конечной периодической частью. Получили противоречие с выбором контрпримера. Лемма доказана.

Лемма 10. Пусть $F$ и $M$ - различные бесконечные максимальные почти слойно конечные подгруппы $G, R(F)$ и $R(M)-$ их слойно конечные радикалы. Тогда

$$
R(F) \cap R(M)=1 \text {. }
$$

Доказательство. Пусть $b \in R(F) \cap R(M), b \neq 1$. Если $R(F) \cap R(M)$ имеет конечные индексы в $F$ и $M$, то по предложению 20 и лемме 9 получаем противоречие с максимальностью $F$ (по лемме Дицмана в почти слойно конечной группе всегда найдется конечная характеристическая подгруппа).

Пусть тогда для одной из подгрупп, например, для $M$, справедливо соотношение $|M: R(M) \cap R(F)|=\infty$. Подгруппа $C=C_{G}(b)$ ввиду свойств слойно конечных групп пересекается с $F$ и $M$ по подгруппам конечного индекса. По лемме $9 C$ - собственная 
подгруппа в $G$. Она обладает почти слойно конечной частью по условию теоремы. Слойно конечный радикал $R(C)$ группы $C$, очевидно, также пересекается с $F$ и $M$ по подгруппам конечного индекса. Так как $|M: R(C) \cap M|<\infty$, а $|M: R(F) \cap M|=\infty$, радикал $R(C)$ не лежит в $F$. Ввиду слойной конечности группы $R(C)$ в ней найдется подгруппа $B$ такая, что $F \cap R(C)=F \cap B$ и $|B: F \cap B|<\infty$. По предложению 20 существует подгруппа $T \leqslant B \cap F$, в нормализатор которой входят подгруппы $F$ и $B$. Из-за максимальности подгруппы $F$ получаем противоречие с почти слойной конечностью периодической части нормализатора $N_{G}(T)$. Лемма доказана.

По предложению 18 все периодические абелевы подгруппы группы $G$ не могут быть черниковскими, так как в этом случае сама группа $G$ обладала бы черниковской и, значит, почти слойно конечной периодической частью. Тогда, не нарушая общности рассуждений, будем считать, что в группе $G$ имеется собственная нечерниковская почти слойно конечная подгруппа. По лемме Цорна и лемме 9 среди всех собственных нечерниковских почти слойно конечных подгрупп группы $G$ найдется максимальная. Зафиксируем за этой подгруппой в дальнейшем обозначение $H$. Через $M$ будем обозначать нормализатор группы $H$ в группе $G$. Очевидно, периодическая часть группы $M$ совпадает с $H$, и группа $M$ является максимальной подгруппой в $G$, обладающей почти слойно конечной периодической частью и содержащей $H$. Ввиду выбора контрпримера $G \neq H$.

Лемма 11. Если для некоторого элемента а конечного порядка пересечение $C_{G}(a) \cap H$ бесконечно, то $C_{G}($ a) содержится в $M$.

Доказательство. Предположим, что найдется элемент $а$ конечного порядка, для которого $\left|C_{G}(a) \cap H\right|=\infty$. Очевидно, что пересечение $C_{G}(a) \cap R(H)=D$ бесконечно. Включим $C_{G}(a)$ в максимальную подгруппу с почти слойно конечной периодической частью $B$. Тогда $D \leqslant Y \cap B$. Так как $|B: R(B)|<\infty$, индекс $|D: R(B) \cap D|$ конечен. Тогда $|D: R(B)|$ нетривиально.

Следовательно, пересечение $R(H) \cap R(B)$ так же нетривиально, и по лемме $10 B=H$. Но тогда $C_{G}(a) \leqslant M$. Лемма доказана.

\section{Јемма 12. Подгруппа Н почти нильпотентна.}

Доказательство. Предположим, что лемма неверна и централизаторы всех элементов простых порядков из $H$ бесконечны в $H$.

Пусть $a-$ некоторый элемент простого порядка из $H$. Группа $L_{s}=\operatorname{gr}\left(a, s^{-1} a s\right)$ конечна для любого элемента $s \in G \backslash M$ ввиду того, что группа $G$ является группой Шункова. Обозначим пересечение $L_{s} \cap M$ через $H_{s}$ и предположим, что $H_{s} \cap H_{s}^{g}$ содержит неединичный элемент $b$ простого порядка для некоторого элемента $g$ из $L_{s} \backslash H_{s}$. Очевидно, что элемент $b$ попадет в $H$, и согласно предположению и лемме $11 C_{G}(b) \leqslant M$.

В то же время $b \in H_{s}^{g}$, и значит, $B \in M^{g}$. Аналогично получаем, что $C_{G}(b) \leqslant g^{-1} M g$. Тогда $S_{G}(b) \leqslant M \cap g^{-1} M g$ и периодическая часть $C$ группы $C_{G}(b)$ содержится в пересечении периодических частей $H$ и $H^{g}$ групп $M$ и $M^{g}$.

Поскольку группы $H$ и $H^{g}$ почти слойно конечны, индексы $|C: C \cap R(H)|$ и $\left|C: C \cap R\left(H^{g}\right)\right|$ конечны. Тогда $R(H) \cap R\left(H^{g}\right)$ обладает неединичным элементом и по лемме 10 справедливо равенство $H=H^{g}$ вопреки выбору элемента $g$.

Противоречие означает, что $L_{s}, H_{s}$ составляют пару Фробениуса и сама группа $L_{s}$ является группой Фробениуса с ядром $F_{s}$ и дополнением $H_{s}$, содержащим $a$. По свойствам групп Фробениуса элемент $a^{s}$ сопряжен с $a$ при помощи некоторого элемента $f$ из $F_{s}$. Следовательно,

$$
L_{s}=\operatorname{gr}\left(a, s^{-1} a s\right)=\operatorname{gr}\left(a, f^{-1} a f\right)=\operatorname{gr}(a, f),
$$


и тогда видим, что $L_{s}=F_{s} \lambda(a)$, то есть подгруппа (a) является неинвариантным множителем группы Фробениуса $L_{s}$.

По предложению $19 G=F \lambda N_{G}((a))$ и $F \lambda(a)$ - группа Фробениуса с нетривиальным ядром $F$ и дополнением (a). Для произвольного элемента $f$ из $F$ подгруппа $\operatorname{gr}(a, f)$ является подгруппой группы Фробениуса $F \lambda(a)$. Тогда из свойств групп Фробениуса следует, что $a f=b^{-1} a b$ для некоторого элемента $b$ из $F$. Отсюда и из равенства

$$
\operatorname{gr}(a, f)=\operatorname{gr}(a, a f)=\operatorname{gr}\left(a, b^{-1} a b\right)
$$

следует справедливость включения $f \in g r\left(a, b^{-1} a b\right)$, что влечет конечность порядка элемента $f$. Таким образом, $F$ - периодическая группа, и по предложению 17 она обладает нетривиальным центром. Тогда по условиям теоремы группа $F$ должна быть локально конечной, а это противоречит лемме 9. Теперь справедливость леммы очевидным образом вытекает из предложения 16.

Лемма 13. В группе $H$ найдется лишь конечное число классов сопряженных элементов простого порядка, имеючих конечные чентрализаторы в $H$.

Доказательство. Так как $H$ почти слойно конечна, представим ее в виде произведения $H=R(H) K$, где $R(H)$ - слойно конечный радикал группы $H, K$ - ее конечная подгруппа. Простые делители порядков из $H \backslash R(H)$ делят $|H: R(H)|$ и являются подмножеством $\pi(K)$. Все элементы из $R(H)$ по лемме Дицмана обладают бесконечными централизаторами. Поэтому почти регулярные элементы простых порядков находятся в $H \backslash R(H)$.

Пусть $r$ - элемент простого порядка $q$ из $H$ с конечным централизатором $C_{H}(r)$. Рассмотрим содержащую его силовскую $q$-подгруппу $Q$ из $H$. По лемме $8 Q$ - черниковская группа. Если она конечна, то в ней число элементов простого порядка конечно. Пусть она бесконечна. Ввиду строения черниковской $q$-группы (см. предложение 21 ) в ней число несопряженных элементов простого порядка $q$ с конечными централизаторами конечно. Тогда по предложению 13 число таких элементов в $H$ также конечно. Теперь, замечая, что $\pi(K)$ - конечное множество, получаем утверждение леммы.

Лемма 14. $B$ Н найдется элемент простого порядка с бесконечным централизатором в $H$, в котором нет элементов с конечными централизаторами в $H$.

Доказательство. По лемме 13 множество $h_{1}, h_{2}, \ldots, h_{l}$ всех несопряженных элементов с конечными централизаторами из $H$ конечно. Значит,

$$
\mu=\bigcup_{i=1}^{l} \pi\left(C_{H}\left(h_{i}\right)\right)
$$

- конечное множество. Тогда мы можем выбрать элемент такого достаточно большого порядка $p \in \pi(H)$ (множество $\pi(H)$ бесконечно, так как $H-$ нечерниковская почти слойно конечная группа), что $p \notin \mathcal{M}$. Очевидно, это и будет искомый элемент. Лемма доказана.

Замечание 1. В силу строения нечерниковской почти слойно конечной группы $H$ будем считать, не нарушая общности рассуждений, что число $р$ выбрано так, что оно не делит индексы $|H: R(H)|$ и $|H: L(H)|$, где $R(H)$ - слойно конечный радикал, а $L(H)-$ нильпотентный радикал группы $H$. 
В дальнейшем будем рассуждать об элементе $a$ из $H$ простого порядка, который удовлетворяет лемме 14, а его порядок выбран согласно замечанию 1.

Лемма 15. Простое число р можно выбрать таким достаточно большим, что силовские p-подгруппь в $L_{g}=\operatorname{gr}\left(a, a^{g}\right), g \in G \backslash M$, ииклические.

Доказательство. Рассмотрим подгруппы вида $L_{g}=\operatorname{gr}\left(a, a^{g}\right), g \in G \backslash M$. Так как $G$ является группой Шункова, подгруппы $L_{g}$ конечны. Обозначим через $S$ силовскую $p$ подгруппу из $L_{g}$, содержащую элемент $a$. Так как $S$, будучи конечной $p$-группой, обладает нетривиальным центром, выбираем элемент $b$ простого порядка из $Z(S)$. Ввиду выбора элемента $a$ централизатор $C_{H}(b)$ бесконечен. Тогда по лемме $11 C_{G}(b) \leqslant H$. Следовательно, $S$ лежит в $H$. Пусть $S$ не является циклической подгруппой. Обозначим элементарную абелеву подгруппу порядка $p^{2}$ из $S$, содержащую элемент $a$, через $R$.

Рассмотрим подгруппу $O_{p^{\prime}}\left(L_{g}\right) 入 R$, ввиду теоремы Файта-Томпсона $O_{p^{\prime}}\left(L_{g}\right) \neq 1$. Согласно предложению 15

$$
O_{p}^{\prime}\left(L_{g}\right)<\operatorname{gr}\left(C_{g}(r) \mid r \in R^{\#}\right)
$$

Как отмечалось выше, элементы $r$ из $R^{\#}$ имеют бесконечные централизаторы в $H$ и в силу леммы 11 лежат в $H$ вместе с $O_{p^{\prime}}\left(L_{g}\right)$. Подгруппа $R$ лежит в $H$ ввиду включения $R<C_{G}(a)$. Таким образом, $O_{p^{\prime}}\left(L_{g}\right) \lambda R<H$.

Ввиду выбора $p$ подгруппа $R$ содержится в нильпотентном радикале $L(H)$ подгруппы $H$. Пусть $Q-$ силовская $q$-подгруппа из $O_{p^{\prime}}\left(L_{g}\right)$. По лемме Фраттини $Q$ выберем таким образом, чтобы $Q$ нормализовалось подгруппой $R$. Если $Q<L(H)$, то, очевидно, $Q \times R$. Если $q$ - делитель индекса $|H: L(H)|$, то по определению нильпотентного радикала $R$ нормализуется подгруппой $Q$. Таким образом, опять получаем $Q \times R$. Так как это рассуждение проходит для любого $q \in \pi\left(O_{p^{\prime}}\left(L_{g}\right)\right)$, заключаем, что $O_{p^{\prime}}\left(L_{g}\right)<C_{G}(a)$. Отсюда и ввиду выбора элемента $a$ все элементы из $O_{p^{\prime}}\left(L_{g}\right)$ имеют в $H$ бесконечные централизаторы, по лемме 11 целиком лежащие в $H$. Фиксируем произвольный элемент $c \neq 1$ из $O_{p^{\prime}}\left(L_{g}\right)$. Как показано выше, $a \in C_{G}(c) \leqslant M$. Проведя аналогичные рассуждения относительно подгруппы $M^{g}$ вместо $M$ и элемента $a^{g}$ вместо $a$, видим, что $a^{g} \in C_{G}(c) \leqslant M^{g}$. Значит, по леммам 10 и $14 H=H^{g}$ для некоторого элемента $g \in G \backslash M$. Полученное противоречие доказывает лемму.

Лемма 16. Для любого элемента $g \in G \backslash M$ группа вида $L_{g}=\operatorname{gr}\left(a, a^{g}\right)$ не содержится 6 $M$.

Доказательство. Предположим, что лемма неверна и нашелся такой элемент $g \in G \backslash M$, что $L_{g}=\operatorname{gr}\left(a, a^{g}\right)<M$. Тогда согласно замечанию 1 элементы $a, a^{g}$ содержатся в слойно конечном радикале группе $H$, и централизаторы $C_{H}(a)$ и $C_{H}\left(a^{g}\right)$ бесконечны. Тогда бесконечен и централизатор $C_{H^{-1}}(a)$. По лемме $10 H=H^{g^{-1}}$. Получили противоречие с выбором элемента $g$. Лемма доказана.

Лемма 17. В почти слойно конечной группе $M$ существует лишь конечное число несопряженных конечных разрешимых подгрупп заданного порядка.

Доказательство. Для черниковской группы утверждение леммы следует из предложения 23. Пусть $M$ - нечерниковская группа.

Пусть сначала в группе $M$ найдется бесконечное множество элементарных абелевых $q$-подгрупп порядка $k$

$$
L_{1}, L_{2}, \ldots, L_{n}, \ldots
$$


Включим групту $L_{n}$ в силовскую $q$-подгруппу $Q_{n}$ из $M, n=1,2, \ldots$ По лемме 8 силовские примарные подгруппы в $M$ черниковские, поэтому можем применить к $M$ предложение 13 , по которому все силовские $q$-подгруппы $Q_{1}, Q_{2}, \ldots, Q_{n}, \ldots$ сопряжены в $M$. Теперь по предложению 23 внутри $Q_{n}, n=1,2, \ldots$, найдется лишь конечное число несопряженных подгрупп порядка $|L|$, и для элементарных абелевых подгрупп утверждение леммы доказано.

Пусть теперь

$$
L=L_{1}, L_{2}, \ldots, L_{n}, \ldots
$$

- последовательность разрешимых подгрупп заданного порядка $k$. Доказательство будем вести индукщией по $k$. Так как все подгруппы последовательности разрешимы, они обладают нормальными элементарными абелевыми подгруппами

$$
Q=Q_{1}, Q_{2}, \ldots, Q_{n}, \ldots
$$

соответственно. По доказанному выше среди них лишь конечное число несопряженных в $M$. Не нарушая общности рассуждений, будем считать, что все они сопряжены с $Q$, то есть

$$
Q_{n}^{c_{n}}=Q, \quad c_{n} \in M, \quad n=1,2, \ldots
$$

Рассмотрим $A=N_{H}(Q)$. Очевидно, что $L_{n}^{c_{n}}<A,\left|L_{n}^{c_{n}} / Q\right|<k$. Так как класс почти слойно конечных подгрупп, очевидно, замкнут относительно взятия факторгрупп, то $A / Q$ - почти слойно конечная группа, и по индуктивному предположению среди

$$
L_{1}^{c_{1}} / Q, L_{2}^{c_{2}} / Q, \ldots, L_{n}^{c_{n}} / Q \ldots
$$

только конечное число несопряженных в $A / Q$ подгрупп. Но тогда такое же утверждение верно и для подгрупп начальной последовательности. Лемма доказана.

Лемма 18. Множество классов сопряженных элементарных абелевых подгрупп из $H c$ конечными чентрализаторами в $Н$ конечно.

Доказательство. Ввиду того, что в слойно конечной группе централизатор любого элемента имеет конечный индекс, нам достаточно рассмотреть только элементарные абелевы $q$-подгруппы для $q \in \pi=\pi(H \backslash R(H))$. Поскольку $\pi$ - конечное множество, а порядки элементарных абелевых $q$-подгрупп из $H$ не могут расти неограниченно для каждого $q$ из $\pi$, имеется только конечное число вариантов для порядков таких подгрупп. Отсюда по лемме 17 получаем утверждение леммы.

Замечание 2. В дополнение к выбору числа $p$ согласно лемме 18 можем считать, что оно не делит порядки элементов из множества

$$
\bigcup_{K \in H} \pi\left(C_{H}(K)\right)
$$

где $K$ пробегает все элементарные абелевы подгруппы из $H$, имеющие в $H$ конечные централизаторы (это множество по лемме 18 конечно, а $\pi(H)$ бесконечно в силу выбора $H)$.

Завершим доказательство теоремы 2. В силу леммы 15 считаем, не нарушая общности рассуждений, что найдется элемент $a \in H$ порядка $p$ такого, что силовская $p$-подгруппа в 
$L_{g}=\operatorname{gr}\left(a, a^{g}\right), g \in G \backslash M$, циклическая. Рассмотрим факторгруппу $L_{g} / O_{p^{\prime}}\left(L_{g}\right)$. Очевидно, она имеет порядок $p$ и, следовательно, $L_{g}=O_{p^{\prime}}\left(L_{g}\right) \lambda(a)$.

Если для всех $g \in G \backslash M$ элемент $a$ действует регулярно на нильпотентном радикале $N_{g}$ групшы $L_{g}=\operatorname{gr}\left(a, a^{g}\right)$, то по предложению 14 он действует регулярно на $O_{p^{\prime}}\left(L_{g}\right)$. В этом случае все $L_{g}$ являются группами Фробениуса с неинвариантным множителем $(a)$. Применяя, как и в конце доказательства леммы 12, предложения 17 и 19, получаем, что $G$ обладает нетривиальной нормальной локально конечной подгруппой вопреки лемме 9 .

Значит, остается предположить, что для некоторого элемента $g \in G \backslash M$ в группе $L_{g}=\operatorname{gr}\left(a, a^{g}\right)$ элемент $a$ перестановочен с некоторым неединичным элементом $b$ из нильпотентного радикала $N_{g}$ группы $L_{g}$. Рассмотрим пересечение $D_{g}=N_{g} \cap H$. Так как $b \in D_{g}$, это пересечение нетривиально.

Как и в лемме 15 , покажем, что из $D_{g} \lambda(a)<H$ вытекает, что $D_{g} \times(a)$. Нильпотентная группа $D_{g}$, очевидно, содержит нетривиальную нормальную элементарную абелеву подгруппу $A_{g}$. Группа $A_{g}$ имеет бесконечный централизатор в группе $H$ согласно замечанию 2. Отсюда, по лемме $10 C_{g}\left(A_{g}\right) \leqslant M$. Включим нормализатор подгруппы $A_{g}$ в максимальную подгруппу $W$ группы $G$, обладающей почти слойно конечной периодической частью. Ввиду того, что пересечение $H \cap W$ содержит бесконечную подгруппу $C_{H}\left(A_{g}\right)$ в силу леммы 10 справедливо равенство $M=W$. Отсюда следует включение $N_{G}\left(A_{g}\right) \leqslant M$, и значит, $N_{G}\left(D_{g}\right) \leqslant M$. Если $N_{g} \neq D_{g}$, то ввиду нормализаторного условия в нильпотентных группах нормализатор подгруппы $D_{g}$ в $N_{g}$ отличен от $D_{g}$ и по доказанному лежит в $H$. Получаем противоречие с построением $D_{g}$.

Если же $N_{g}=D_{g}$, то ввиду нормальности $N_{g}$ в $L_{g}$ и включения $N_{G}\left(D_{g}\right) \leqslant M$ получаем, что $L_{g}<H$ вопреки лемме 16 . Теорема доказана.

\section{Список литературы}

1. Сенашов В. И., Группы с условием минимальности для не почти слойно конечных групп. Укр. матем. ж. (1991) 43, №7-8, 1002-1008.

2. Сенашов В. И., Почти слойная конечность периодической группы без инволюций. Укр. матем. ж. (1999) 51, №11, 1529-1533.

3. Адян С. И., Проблема Берисайда и тождества в группах. Наука, Москва, 1975.

4. Ольшанский А. И., Геометрия определяющих соотиошений в группе. Наука, Москва, 1989.

5. Черников С. Н., Группы с заданными свойствами системы подгрупп. Наука, Москва, 1980.

6. Каргаполов М. И., Мерзляков Ю. И., Осиовы теории групп. Наука, Москва, 1982.

7. Мерзляков Ю. И., Матричное представление групп внешних автоморфизмов черниковских групп. Алгебра и логика (1969) 8, №4, 478-482.

8. Шунков В. П., О локально-конечной группе с экстремальными силовскими р-подгруппами по некоторому простому числу р. Сиб. матем. ж. (1967) 8, №1, 213-229.

9. Курош А. Г., Теория групn. Наука, Москва, 1967.

10. Сенашов В. И., Слойно копечиые группы. Наука, Новосибирск, 1993.

11. Blackburn N., Some remarks on Chernikov's p-groups. J. Math. (1962) 6, 421-433.

12. Каргаполов М. И., Локально конечные группы, обладающие нормальными системами с конечными факторами. Сиб. матем. ж. (1961) 2, №6, 853-873.

13. Павлюк И. И., Шунков В. П., О локально конечных группах с условием $\min -p$ по всем $p$. VII Всесоюзный симпозиум по теории групп, Красноярск, 1980, 84-85. 
14. Горчаков Ю. М., О существовании абелевых подгрупп бесконечного ранга в локально разрешимых группах. Докл. АН СССР (1964) 156, №1, 17-20.

15. Каргаполов М. И., Некоторые вопросы теории нильпотентных и разрешимых групп. Докл. АН CССР (1959) 127, №6, 1164-1166.

16. Gorenstein D., Finite groups. Harper and Row, New York, 1968.

17. Бусаркин В. М., Горчаков Ю. М., Конечиые расщепляемые группы. Наука, Москва, 1968.

18. Шунков В. П., О локально конечных группах конечного ранга. Алгебра и логика (1971) 10, №12, 199-225.

19. Шунков В. П., Mp-группьl. Наука, Москва, 1990.

20. Brauer R., Some applications of theory of block of characters of finite groups. II. J. Algebra (1964) 1, 307-334.

21. Хухро Е. И., Нильпотентные периодические группы с почти регулярным автоморфизмом простого порядка. Алгебра и логика (1987) 26, №4, 502-517.

22. Шунков В. П., О вложении примариых элементов в группе. Наука, Новосибирск, 1992.

23. Сучкова Н. Г., О существовании периодической части в некоторых бесконечных группах. Teзисы докл. XV Межрегиональной научно-техи. конф. Красноярская гос. архитектурно-строительная академия, Красноярск, 1997, 13-14.

24. Созутов А. И., Шунков В. П., О бесконечных группах, насыщенных фробениусовыми подгруппами. Алгебра и логика (1977) 16, №6, 711-735; (1979) 18, №2, 206-223.

25. Черников Н. С., О бесконечно простых локально конечных группах. Препринт 82.37 , Институт матем. АН УССР, Киев, 1982.

Статья поступила 07.06.2001. 